\title{
SUPERPARAMAGNETIC GRAINS AS SOURCE OF GIANT MAGNETORESISTANCE EFFECT IN DISCONTINUOUS Co/Ag MULTILAYERS
}

\author{
T. Luciński, F. Stobiecki, J. Dubowik \\ Institute of Molecular Physics, Polish Academy of Sciences \\ Smoluchowskiego 17, 60-179 Poznan, Poland \\ AND R. CZAJKa \\ Institute of Physics, Poznań University of Technology \\ Piotrowo 3, 60-965 Poznań, Poland
}

\begin{abstract}
The as-deposited and annealed $\mathrm{Co} / \mathrm{Ag}$ discontinuous multilayer-like structures were examined. Discontinuous Co sublayers are thought to consist of fine superparamagnetic and ferromagnetic grains. Using the bimodal superparamagnetic grain sizes distribution the GMR $(H)$ dependencies were successfully modelled with the use of two Langevin functions simulating the magnetization of the superparamagnetic grains.
\end{abstract}

PACS numbers: 75.70.Cn

Recently there has been a great deal of interest in the influence of annealing of multilayers (Ml) consisting of stacking of very thin ferromagnetic layers (e.g. Co) and layers of an immiscible nonmagnetic elements like $\mathrm{Ag}$ [1]. The annealing of such structures can lead to the formation of magnetic precipitates in a nonmagnetic matrix.

The wedged Co/Ag Mls with nominal sublayer thicknesses of $d_{\mathrm{Co}}=0.26 \mathrm{~nm}$ and $0.65 \mathrm{~nm} \leq d_{\mathrm{Ag}} \leq 1.27 \mathrm{~nm}$, were deposited onto glass substrates using facing target sputtering method. The magnetoresistance $R(H)$ and the magnetization loops $M(H)$ were measured at room temperature in a magnetic field range $\pm 25 \mathrm{kOe}$ for parallel configuration. Both $M(H)$ and $\operatorname{GMR}(H)$ measurements were performed in as-deposited state and after sequential annealing steps at $250^{\circ} \mathrm{C}$ and $300^{\circ} \mathrm{C}$; each step lasting 1 hour. We define the field dependence variation in GMR as $\operatorname{GMR}(H)=[R(H)-R(H=0)] / R(H=0)$.

Figure 1 presents the dependence on $\mathrm{Ag}$ volume concentration $\left(x_{\mathrm{v}}\right)$ of GMR amplitude for as-deposited and annealed Mls. It is seen that the maximum values of GMR occurs for $x_{\mathrm{v}} \approx 0.80$ reflecting the granularity of the Mls. Such a granular structure was confirmed by our scanning tunnelling microscopy (STM) experiment; the grain sizes ranging from $2 \mathrm{~nm}$ to $15 \mathrm{~nm}$ were observed. 


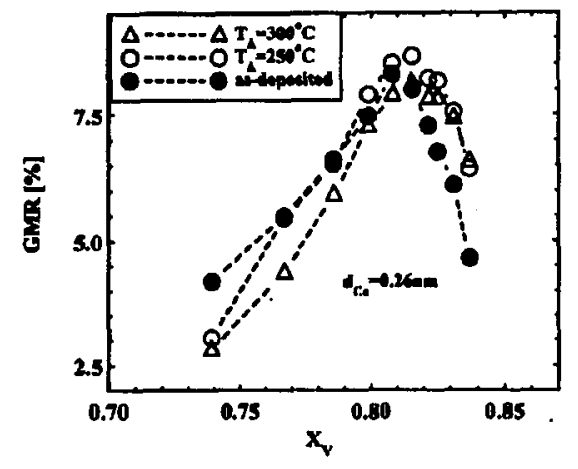

Fig. 1. The Ag volume concentration $x_{\mathrm{v}}$ dependencies of GMR amplitude for as-deposited and annealed Co/Ag Mls.
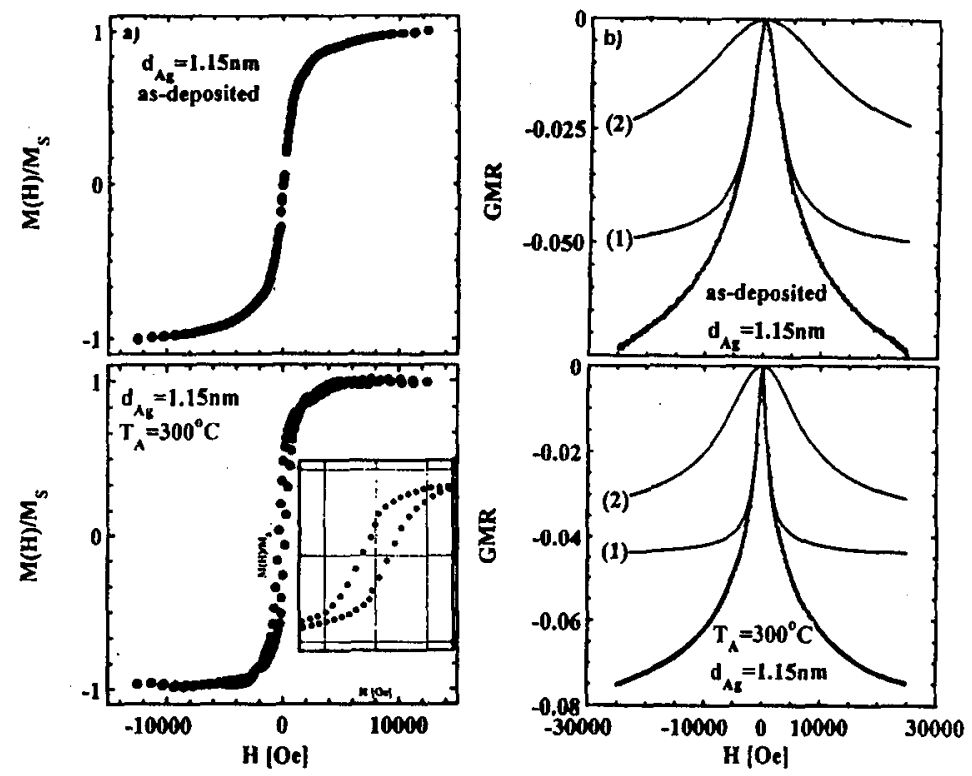

Fig. 2. (a) The normalised magnetization loops for as-deposited and annealed at $T_{\mathrm{A}}=$ $300^{\circ} \mathrm{C}$ Ml. (b) The GMR $(H)$ dependencies for the same $\mathrm{Ml}$ as in (a). The results of fitting (solid lines through the data points) $\operatorname{GMR}(H)$ data with a model described in the text are also shown. Solid lines labelled with (1) and (2) represent decomposition of GMR $(H)$ curves due to the postulated bimodal superparamagnetic grain size distribution.

Figure 2a displays the examples of the normalised $M(H)$ loops for as-deposited and annealed $\mathrm{Ml}$ with $d_{\mathrm{Ag}}=1.15 \mathrm{~nm}$. Except the case of an as-deposited sample where a superparamagnetic $M(H)$ dependence was found, all the Mls were characteristic of ferromagnetically (FM) coupled or uncoupled Mls with distin- 
guishable $H_{\mathrm{C}}$ and $M_{\mathrm{S}}$, where $H_{\mathrm{C}}$ and $M_{\mathrm{S}}$ denote coercive field and saturation magnetization, respectively.

Figure $2 \mathrm{~b}$ shows the GMR $(H)$ dependencies for the same sample as in Fig. 2a. Comparing both panels of Fig. 2 it is evident that the $M(H)$ curves saturate at relatively low magnetic fields whereas $\operatorname{GMR}(H)$ show no sign of saturation. This obvious inconsistency strongly suggests that the origin of GMR in our samples is due neither to the existence of antiferromagnetic (AF) coupling nor to uncorrelated magnetic moments of Co grains. In the latter case GMR $(H)$ should be peaked in $H= \pm H_{\mathrm{C}}$ which is not observed. The specific field dependencies of GMR (i.e. Langevin-like) strongly suggest that the most likely responsible for GMR $(H)$ behaviour in examined MLs are the superparamagnetic (SP) grains. Since the $M(H)$ dependence reflects the net magnetization of Co/ $\mathrm{Ag}$ Mls, then $M(H)$ can be written as $M(H)=M_{\text {ferro }}(H)+M_{\mathrm{SP}}(H)$, where $M_{\mathrm{SP}}(H)$ and $M_{\text {ferro }}(H)$ denote the superparamagnetic and ferromagnetic parts of $M(H)$, respectively. It seems that GMR in our samples is due to the assemblage of SP grains and the ferromagnetic grains are only noticeable in the $M(H)$ behaviour. The existence of the SP component in our Mls was confirmed by zero field cooled (ZFC) and field cooled (FC) magnetization measurements.

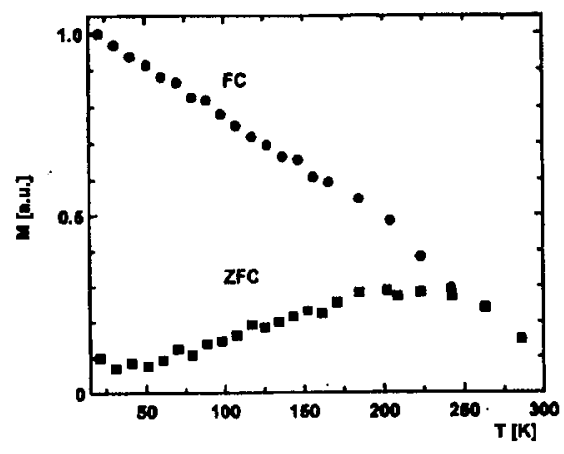

Fig. 3. The ZFC and FC magnetization curves for annealed at $T_{\mathrm{A}}=300^{\circ} \mathrm{C}$ sample with $d_{\mathrm{Ag}}=1.15 \mathrm{~nm}$.

Figure 3 shows the representative ZFC and FC curves for annealed sample with $d_{\mathrm{Ag}}=1.15 \mathrm{~nm}$. A broad maximum near $200 \mathrm{~K}$ is associated with the distribution of blocking temperatures $T_{B}$ which is strictly related with the distribution of the sizes and/or shapes of the SP grains. The upper limit of this distribution, which corresponds to the largest grains, is given by the temperature at which the ZFC curve becomes reversible $(\approx 250 \mathrm{~K})$. A well known equation [2] relates the volume $V$ of the SP grain to the blocking temperature: $k T_{\mathrm{B}} \approx 25 C V$, where $C$ is the total magnetic anisotropy energy density of the grain. Using the hcp Co magnetic anisotropy $K=4.5 \times 10^{5} \mathrm{~J} / \mathrm{m}^{3}$ instead of $C$ we can estimate the average grain size $D$. Assuming a spherical shape of the grains we found $D \approx 6.6 \mathrm{~nm}$. Above $T_{\mathrm{B}}$, in the SP state the $M_{\mathrm{SP}}(H)$ dependencies can be described by the Langevin 
function $L(\alpha)$, where $\alpha=\mu H / k T$ and $\mu$ denotes the average moment of the superparamagnetic grain. Assuming a bimodal SP grain size (BSPGS) distribution the $M_{\mathrm{SP}}(H)$ can be written as a sum of two Langevin functions with different magnetic moments per individual grain. Hence $M_{\mathrm{SP}}(H)=f L\left(\alpha_{1}\right)+(1-f) L\left(\alpha_{2}\right)$, where $f$ is a fraction of the grains bearing the moment $\mu_{1}$. Thus, GMR $(H)$ can be modelled as $\operatorname{GMR}(H)=-A\left[M_{\mathrm{SP}}(H) / M_{\mathrm{S}}\right]^{2}$, where $A$ denotes the GMR amplitude and $\mu_{1,2}$ and $f$ are taken as adjustable parameters. In our model the large grains (1) are thus responsible for low field behaviour in $\operatorname{GMR}(H)$ while the small ones (2) are responsible for slow saturation in high magnetic fields. The results of fitting $\operatorname{GMR}(H)$ with the use of the above equation are shown in Fig. $2 \mathrm{~b}$ (solid lines through the data points). Figure $2 \mathrm{~b}$ shows also the decomposition of $\operatorname{GMR}(H)$ curves due to the postulated BSPGS distribution (solid lines denoted 1 and 2). The quality of the fit, as can be seen from the figures, is excellent. From the values of parameters $\mu_{1,2}$ and under the assumed spherical shape of the grains their diameters $D_{1}$ and $D_{2}$ can be estimated. The value $D_{1} \approx 6 \mathrm{~nm}$ was found for annealed samples with $x_{\mathrm{v}} \approx 0.80$. This grain diameter is in a very good agreement with $D \approx 6.6 \mathrm{~nm}$. The $D_{2}$ values were found to lie between $2 \mathrm{~nm}$ and $2.5 \mathrm{~nm}$, independently of annealing temperature and $d_{\mathrm{Ag}}$. The above discussion and the experimental data support our statement that in our discontinuous $\mathrm{Co} / \mathrm{Ag}$ Mls the assemblage of SP grains is responsible for the occurrence of the GMR effect and for related specific magnetic field dependencies.

\section{Acknowledgment}

This work was financed in part by DPB 62129 P.U.T.

\section{References}

[1] E.A.M. van Alphen, W.J.M. de Jonge, Phys. Rev. B 51, 8182 (1995).

[2] B.D. Cullity, Introduction to Magnetic Materials, Addison-Wesley, Reading 1972, p. 410. 